\title{
X-ray Grating Observations of Recent Recurrent Novae
}

\section{Marina Orio ${ }^{1,2}$, Ehud Behar ${ }^{3}$, J. Gallagher ${ }^{2}$, A. Bianchini ${ }^{4}$, E. Chiosi ${ }^{1}$, J. Luna ${ }^{5}$, T. Nelson ${ }^{6}$, and T. Rauch ${ }^{7}$}

\author{
${ }^{1}$ INAF, Osservatorio Astronomico di Padova, vicolo Osservatorio 5, I-35122 Padova, Italy \\ email: marina.orio@oapd.inaf.it \\ ${ }^{2}$ Dept. of Astronomy, 475 N. Charter Str., Madison WI 53706 \\ ${ }^{3}$ Department of Physics, Technion, Kiryat Hatechnion, Haifa, Israel \\ ${ }^{4}$ Astronomy Department, Padova University, vicolo dell' Osservatorio 3, I-35122 Padova, Italy \\ ${ }^{5}$ Instituto de Ciencias Astronómicas, de la Tierra y del Espacio (ICATE/FCEFyN), \\ Av.España Sur 1512, J5402DSP, San Juan, Argentina \\ ${ }^{6}$ School of Physics and Astronomy, University of Minnesota, \\ 116 Church St SE, Minneapolis, MN 55455 \\ ${ }^{7}$ Institute for Astronomy \& Astrophysics, Kepler Center for Astro \& Particle Physics, \\ Eberhard Karls University, Sand 1, 72076 Tübingen, Germany
}

\begin{abstract}
Two recurrent novae (RNe) that do not host red giants were observed in outburst at the beginning of 2009 and 2010, respectively. The first nova was LMC 2009a, and the second one was U Scorpii. Nova LMC 2009a was a relatively slow RN, and it was quite luminous both at optical and X-ray wavelengths. U Sco is the fastest nova ever recorded. Its supersoft X-ray phase started a couple of weeks after optical maximum and lasted for about a month, while for Nova LMC 2009a this phase started four months after maximum and lasted for 5 months. For both novae, the first X-ray spectrum taken while the luminous supersoft X-ray source was beginning to emerge is remarkably similar with broad and prominent emission lines of nitrogen and carbon accounting for at least $30 \%$ of the X-ray flux. Blue-shifted absorption and red-shifted emission form apparent P-Cyg profiles. We attribute the emission features to the ejecta and show evidence that they are largely due to collisional ionization. In the case of U Sco the absorption lines were embedded in the emission features after the first observation. We find evidence that in $\mathrm{U}$ Scorpii we were observing the Thomson reflected spectrum at a distance of $\simeq 3 R_{\odot}$ from the white dwarf rather than the atmosphere itself. For both novae, the peak temperature was remarkably high, probably close to $900,000 \mathrm{~K}$ for U Sco, and about $600,000 \mathrm{~K}$ for Nova LMC 2009a. We suggest that these two objects represent different stages of RNe secular evolution.
\end{abstract}

Keywords. stars: novae, cataclysmic variables, winds, outflows, individual (U Scorpii, Nova LMC 2009a), white dwarfs - X-rays: binaries, individual (U Scorpii, Nova LMC 2009a)

\section{Introduction}

Two recurrent novae have caught our attention in the last two and a half year and have been sufficiently luminous to be repeatedly observed with X-ray gratings.

Nova LMC 2009a was observed in outburst by Liller (2009) on 2/5/2009 and was observed in the same position as Nova LMC 1971, and is therefore classified as a recurrent nova. This classification is consistent with the optical spectra, which showed remarkable similarities with other recurrent novae, most notably U Sco at maximum (Orio et al. 2009). It was slow for a recurrent nova, with $t_{3} \simeq 18$ days, and no red giant was observed at the nova's position. Swift detected a 1.19 days periodicity in UV, that was marginally detected also in X-rays. This is thought to be the orbital period of the system. 
U Sco has an orbital period of 1.23 days (interestingly close to the one of Nova LMC 2009a) and belongs to the class of RNe with a near main-sequence or moderately evolved secondary (Schaefer \& Ringwald 1995). The secondary spectrum is not detected at quiescence, but at a late post-outburst phase Anupama \& Dewangan (2000) measured the spectral features of a K2 subgiant. The previous outbursts showed that the ejecta are extremely depleted in hydrogen (Barlow et al. 1981), so the secondary must have lost a significant fraction of its envelope. At quiescence, He II lines are dominant and the hydrogen lines are absent or weak, a fact that has been interpreted as accretion of He-rich material from a H-depleted secondary (e.g. Duerbeck et al. 1993). For this reason, U Sco is very interesting as it may end as a Type Ia supernova without hydrogen-rich material. The short outburst, with decay times by 2 and 3 optical magnitudes of $t_{2}=1.2$ days and $\mathrm{t}_{3}=2.6$ days respectively, and the return to quiescence within 67 days indicate a very massive WD, such that sufficient pressure for a thermonuclear runaway is built up at the base of the envelope even with a small accreted envelope mass (e.g. few $\leqslant 10^{-7} \mathrm{M}_{\odot}$, see Starrfield et al. 1988). This nova is thus particularly interesting as a potential progenitor of a Type Ia supernova (SN Ia) because of the high WD mass and the low hydrogen abundance, consistent with limits in SNe Ia spectra.

Since U Sco has shown outbursts on an almost regular timescale of $10 \pm 2$ years (assuming that a couple of eruptions were missed because they occurred when the nova was behind the sun), Schaefer (2010) was able to predict the last eruption of January of 2010 with an uncertainty of only a year. When the event occurred, a strategy was in place to observe the outburst at all wavelengths.

We present here repeated grating X-ray spectra of the two novae, the only means to probe the effective gravity, chemical composition, and effective temperature of the underlying white dwarf once the photosphere shrinks back to the WD radius (e.g. Nelson et al. 2008 and references therein).

\section{Grating Observations of Nova LMC 2009a}

A luminous supersoft X-ray source was observed with the Swift XRT only 70 days after the optical maximum (Bode et al. 2009), a much longer time than for the previously well-monitored recurrent nova RS Oph where the SSS emerged after the first month (see Nelson et al. 2008). On May 6 of 2009, the nova was observed through the XMM-Newton Director's Discretionary Time (DDT) program (see left panel of Figure 1). The soft Xray flux was due to a hot continuum with absorption features, possibly contributing up to about $80 \%$ of the flux, but was also partially due to prominent emission lines, most notably due to nitrogen (N VI He $\gamma, \mathrm{N}$ VI H $\alpha$ at $24.78 \AA$, the N VI He-like triplet at 28-29 $\AA$ ) and carbon (C VI Ly $\gamma$ at $26.99 \AA$ ). The RGS spectrum is noisy, but we can definitely say that the absorption features appear blue-shifted and the emission red-shifted. We discuss this characteristic below in the context of a similar spectrum of U Sco.

Three subsequent spectra, taken between July and September of 2009 (the last two done by us) show the WD becoming much more luminous, with a peak in August and the beginning of the decay in September (right panel of Figure 1). The emission lines remained, and even additional lines of oxygen (O VIII at $19.97 \AA$ and the O VII He-like triplet at 21.6-22.1 $\AA$ ) were observed. Further monitoring with the Swift XRT showed that the nova was below the Swift XRT detection threshold within two additional months. The peak luminosity in 2009 August was near Eddington and the peak temperature was $650,000-700,000 \mathrm{~K}$ on the same date. 

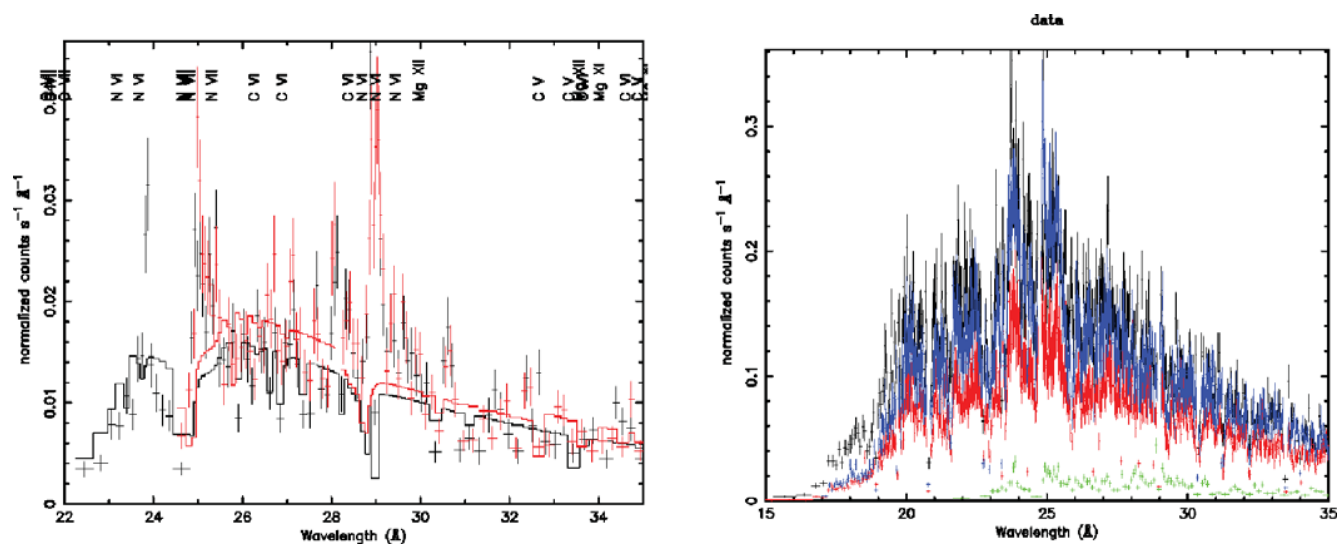

Figure 1. The XMM-Newton RGS spectrum of Nova LMC 2009a on May 6th of 2009 is shown on the left, with an atmospheric model with $\mathrm{T}_{\text {eff }}=600,000 \mathrm{~K}$, while the right panel shows the comparison of all spectra: green, again May; blue, July 20 of 2009; black, August 20 2009; red, September 232009.

\section{Grating Observations of U Scorpii}

U Scorpii was also monitored with Swift, and a Chandra observation was proposed by us with the LETG grating when it became a luminous SSS. XMM-Newton observations were scheduled by Ness et al. (2010) 5 days later, and again after an additional 14 days in the framework of a DDT program.

The Chandra spectrum on day 17 is shown in the left panel of Figure 2. The observation started at orbital phase 0.4463 and covered almost a quarter $(22 \%)$ of the period. We observed a conspicuous continuum between 20 and $35 \AA$, with absorption features of nitrogen, which we attribute to the WD atmosphere. However, about $30 \%$ of the flux was in emission features. The emission lines, seldom observed to be so strong in this wavelength range in most classical novae, in this spectrum are in fact very pronounced and very broad (compared with thermal and instrumental broadening, see e.g. Brinkman et al. 2000, Ness et al. 2005). We attribute the line width to the expansion velocity of the nova shell. The line profiles observed with the LETG are plotted in the left panel of Figure 2 in the velocity space for the two most prominent lines: the Lyman $\alpha \mathrm{H}$-like line of N VII at $24.7792 \AA$ and the He $\alpha$ resonance line at $28.78 \AA$. These profiles appear to be the X-ray equivalent of the optical P-Cyg profile, with red-shifted emission and blue-shifted absorption, often observed in the optical spectra of novae. The right panel of Figure 2 shows that the N VII emission appears blue-shifted by $2,200 \pm 200 \mathrm{~km} \mathrm{~s}^{-1}$ in absorption, and red-shifted by the same amount in emission. The N VI resonance line with rest wavelength $28.78 \AA$ is instead blue-shifted by $2,700 \pm 300 \mathrm{~km} \mathrm{~s}^{-1}$ in absorption, and red-shifted by $1,500 \pm 300 \mathrm{~km} \mathrm{~s}^{-1}$ in emission, so the two components do not cross around zero but towards the red. At optical wavelengths this usually occurs after the P Cyg profile has already been detected for some time, and when it is about to disappear in later spectra. The line profile can be defined perhaps as a "pseudo-P-Cyg" rather than a usual P Cyg, because the absorption features, except for O I at 23.476 ^ which is interstellar, are expected to be produced in the WD atmosphere rather than being formed above it.

The second XMM-Newton RGS spectrum on day 22 after the outburst shows the emergence of oxygen features ( $\operatorname{Ly} \alpha$ and the $\operatorname{He} \beta$ triplet) but is otherwise very similar to the Chandra one (Figure 2). On March 5, however, the WD spectrum is harder and more luminous, while new emission lines also appear in the harder range, all the way to $\mathrm{Ne}$ 

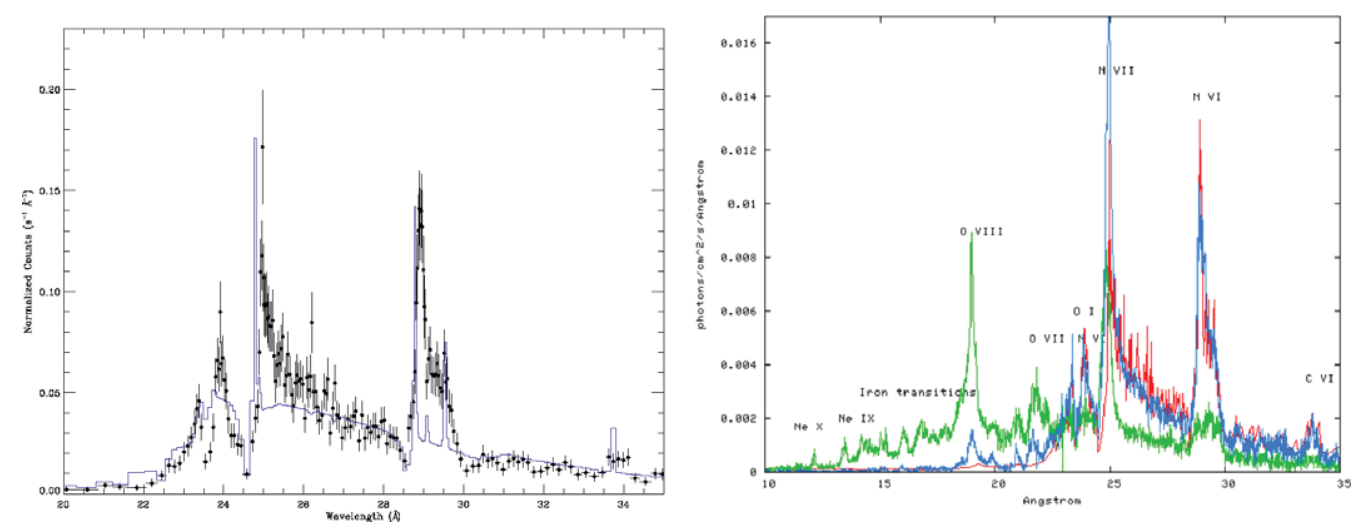

Figure 2. The left panel shows the Chandra spectrum of U Sco on day 17 compared to a model atmosphere with a $600,000 \mathrm{~K} \mathrm{~T}_{\text {eff }}$ and collisional ionization in the ejecta with 10 -fold enhanced helium and nitrogen (without reproducing the velocity broadening). The right panel shows the comparison of all "fluxed" spectra: the Chandra spectrum is displayed in red, the averaged RGS1-RGS2 spectrum of February 2010 is plotted in blue, and the March 2010 RGS1-RGS2 spectrum is in green.

(the Ne X Lyman $\alpha$ line at $12.13 \AA$ ). Moreover, in this third observation the high $\mathrm{S} / \mathrm{N}$ of the EPIC-pn allows us to observe a partial eclipse that is very similar in depth and shape to the total eclipse observed at optical wavelengths during the same days (see Figure 3 ). This eclipse cannot be total because the lines do not vary significantly in flux, while only the continuum does. The size of the object that has been eclipsed has to be similar in dimensions to the size of the disk, which is $3 \mathrm{R}_{\odot}$ (Schaefer et al. 2011). This implies that the source of the continuum cannot be the WD itself, and we infer that we are observing Thomson scattered WD radiation, probably scattered by an an accretion disk corona like in CAL 87 (Orio et al. 2004). The eclipse had already started in the February XMMNewton observation, while at optical wavelengths the eclipse had already been observed, but in February the eclipse was much less deep and exhibited a pronounced oscillation with a semi-regular period of about 3 hours. The absorption features are not measurable in any of the two XMM-Newton observations, but they are embedded in the absorption features, consistently with the shift towards zero velocity of the centroid of the emission lines.

Model atmospheres fits with Rauch's model (see Rauch et al. 2010) indicate that the WD effective temperature was in the range $550,000-750,000 \mathrm{~K}$ in the first and second observation, 800,000 to $1,000,000 \mathrm{~K}$ in the third observation (at maximum). Ad hoc models with fine-tuned abundances would be necessary for a more precise determination of the temperature. In any case, $\log (g) \geqslant 9$ in all models. The high effective gravity and the peak temperature in March indicate a WD mass above $1.2 \mathrm{M}_{\odot}$, consistent with previous estimates.

\section{Conclusions}

Nova LMC 2009a and U Sco are two novae of the non-symbiotic type with different evolutions. In both outbursts, although at different times, the nova shell emits remarkably similar emission lines, which we attribute mostly to collisional ionization in the shell. We would like to suggest that the explanation of collisional ionization is most likely found in colliding ejecta in the same outburst, probably with a faster wind preceded by a slower one. 

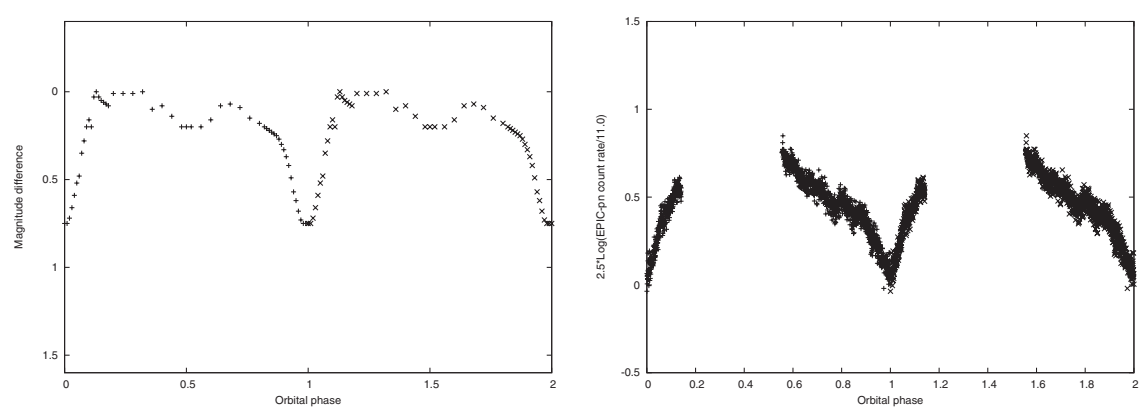

Figure 3. The average optical V magnitude light curve of U Sco during days 32-41, from Schaefer et al., 2011 (left panel), compared with the observed X-ray light curve measured with the EPIC-pn instrument aboard XMM-Newton (right panel). In the right panel, the logarithm of the day 34 count rate is normalized to the minimum value of the measurements, multiplied by 2.5 to match the magnitude scale, and binned in time with $50 \mathrm{~s}$ bins before conversion to phase.

However, the X-ray evolution of the two novae is altogether very different, with a time scale of only 5 weeks for U Sco to reach the maximum WD luminosity, compared with half a year for Nova LMC 2009a. As predicted by the models, the peak temperature of U Sco is higher than for Nova LMC 2009a. Since X-ray turn-off followed the maximum within a short time, and the time to X-ray turnoff of the WD is inversely proportional to the WD mass while the peak temperature is directly proportional to WD mass, this trend confirms the nova models remarkably well.

It is not unlikely that the two novae, with a very close orbital period, are similar systems in different stages of secular evolution. We suggest that the data indicate that the less hot, slower Nova LMC 2009a is in a much earlier secular phase, and both RNe may well be on evolutionary tracks toward SNe Ia explosions.

\section{References}

Anupama, G. C. \& Dewangan, G. C. 2000, AJ, 119, 1359

Barlow, M. J., et al. 1981, MNRAS, 195, 61

Brinkman A. C., et al. 2000, ApJ, 530, L111

Liller, W. 2009, IAUC 9019

Nelson T., et al. 2008, ApJ, 673, 1067

Ness, J.-U., et al. 2005, MNRAS, 364, 1015

Ness, J.-U., et al. 2010, ATel 2477

Nelson, T., Orio, M., et al. 2008, ApJ, 673, 1067

Orio, M.; Ebisawa, K., Heise, J., \& Hartmann, J. 2003, RMXAA, 610, 194

Ness, J-U., Brickhouse, N. S., Drake, J. J., \& Huenemoerder, D. P. 2003, ApJ, 598, 1289

Ness, J-U., Brickhouse, N. S., Drake, J. J., \& Huenemoerder, D. P. 2003, ApJ, 598, 1289

Rauch, T., Orio, M., Gonzales-Riestra, R., Nelson, T., Still, M., Werner, K., \& Wilms, J. 2010, ApJ, 717, 363

Schaefer, B. E. \& Ringwald, F. A. 1995, ApJ, 447, L45

Schaefer, B., et al. 2010, AJ, 140, 925 